\title{
Single-cycle optical control of beam electrons
}

\author{
Yuya Morimoto ${ }^{1, *,+}$ and Peter Baum ${ }^{1,2, *}$ \\ ${ }^{1}$ Ludwig-Maximilians-Universität München, Am Coulombwall 1, 85748 Garching, Germany \\ ${ }^{2}$ University of Konstanz, Universitätsstraße 10, 78457 Konstanz, Germany \\ *Corresponding to: peter.baum@uni-konstanz.de,yuya.morimoto@fau.de \\ +Current address: Friedrich-Alexander-Universität Erlangen-Nürnberg, Staudtstraße 1, 91058 \\ Erlangen, Germany
}

Dated: April 23, 2020

Single-cycle optical pulses with a controlled electromagnetic waveform allow to steer the

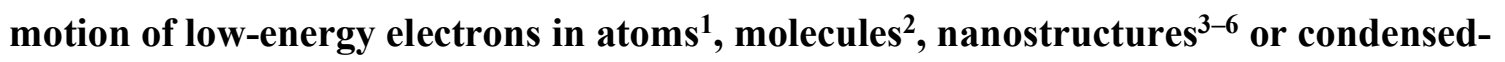
matter $^{7-9}$ on attosecond dimensions in time. However, high-energy electrons under singlecycle light control would be an enabling technology for beam-based attosecond physics with free-electron lasers or electron microscopy. Here we report the control of freely propagating $\mathrm{keV}$ electrons with an isolated optical cycle of infrared light and create a modulated electron current with a peak-cycle-specific sub-femtosecond structure in time. The evident effects of the carrier-envelope phase, amplitude and dispersion of the optical waveform on the temporal composition, pulse durations and chirp of the free-space electron wavefunction demonstrate the sub-cycle nature of our control. These results create novel opportunities in laser-driven particle acceleration ${ }^{10,11}$, seeded free-electron lasers ${ }^{12}$, attosecond space-time imaging ${ }^{13}$, electron quantum optics ${ }^{14}$ and wherever else high-energy electrons are needed with the temporal structure of single-cycle light. 
Modern attosecond science aims at the exploration of ultrafast charge carrier dynamics in complex materials by investigating the time-dependent responses of bound electrons to a single cycle of optical excitation. The electric field as a driving force can for example produce macroscopic electric currents ${ }^{7,8}$ or spin waves ${ }^{9}$ as a consequence of electronic motion on atomic dimensions. While such light-cycle control of low-energy electrons is ideal for ultrafast electronic operations or for the generation of intense attosecond light pulses, a single-cycle control of the temporal, spatial and energetic structure of high-energy electrons beams would be crucial for laser-driven particle accelerators ${ }^{10,11}$, ultrafast electron imaging ${ }^{15,16}$, electron-based quantum information technology ${ }^{17}$ or attosecond science with free-electron lasers ${ }^{12}$. However, so far only radio-frequency fields ${ }^{18-20}$, terahertz radiation ${ }^{21-24}$ or optical multi-cycle pulses ${ }^{10,11,13,25-27}$ have been employed for the acceleration, compression or metrology of free-space electron pulses on time scales of tens of femtoseconds ${ }^{19-21,23,24}$ or in form of multi-pulse sequences ${ }^{10,11,13,25-27}$. Although pioneering electron acceleration experiments were reported with few-cycle laser pulses $^{28-30}$, it remains to be established whether and how an isolated optical field cycle can control the temporal shape of free-space electrons at $\mathrm{keV}$ to $\mathrm{MeV}$ energies with attosecond precision, in order to merge the unprecedented power and brightness of high-energy electron beams with the temporal structure provided by single-cycle laser light.

The sketch and concept of our experiment is shown in Fig. 1a. A femtosecond $\mathrm{Yb}: \mathrm{KGW}$ laser (magenta) is used for generating a beam of $70-\mathrm{keV}$ electron pulses (blue) and for pumping a mid-infrared optical parametric amplifier ${ }^{31}$ for single-cycle waveform generation. Briefly, nearinfrared pulses (orange) from the optical parametric amplifier (NOPA) are mixed with fundamental pulses from the $\mathrm{Yb}: \mathrm{KGW}$ laser (magenta) in a $\mathrm{LiGaS}_{2}$ crystal for differencefrequency generation. Passive phase locking produces mid-infrared pulses with a stable carrierenvelope phase. The spectrum (see Figure $1 \mathrm{~b})$ spans from $26 \mathrm{THz}(11.5 \mu \mathrm{m})$ to $62 \mathrm{THz}(4.8 \mu \mathrm{m})$ at $-20 \mathrm{~dB}$ level, exceeding one optical octave. The temporal waveform is characterized by electro-optical sampling and shown in Fig. $1 \mathrm{c}$. The main field cycle at $t \approx 0$ fs is 1.8 times stronger than the adjacent positive peaks at $t \approx \pm 23$ fs and the pulse shape therefore allows a subcycle control of the electron beam. A split and displaced parabolic mirror (yellow) focuses two such pulses onto ultrathin membranes (green) for temporal modulation of the electron 
wavepacket (blue) and its subsequent streaking characterization. The amplitude and the carrierenvelope phase (CEP) of the two mid-infrared pulses are adjusted independently (see Fig. 1a).

In order to control the electron beam by a single cycle of light (red), we invoke electrontransparent metallic membranes with an extremely broad bandwidth as the modulation elements for photon-electron energy exchange ${ }^{32,33}$. Free-standing silicon nitride membranes are coated with $\sim 10 \mathrm{~nm}$ of aluminium. Although this coating is $>500$ times thinner than a wavelength, we find that the membranes reflect our single-cycle mid-infrared pulses efficiently over the full range of their octave-broad spectrum. The $70-\mathrm{keV}$ electrons pass though the membrane within a time of $<140$ as and are therefore injected into the optical electromagnetic field on the backside within sub-cycle time. This abrupt injection from a field-free region before the membrane into the optical field cycles on the backside causes a time-dependent electron energy modulation that follows the temporal integral of the optical waveform ${ }^{32,34}$. At the first membrane, the one for single-cycle control, we apply an optical peak field strength of $\sim 25 \mathrm{MV} / \mathrm{m}$. Vacuum is a dispersive medium for our electrons and the cycle-induced energy modulation is therefore transformed by propagation into a modulation of current density in time ${ }^{13}$. We characterize the final electron pulse shape at a distance of $\sim 12 \mathrm{~mm}$ at a second metal membrane under illumination of a stronger single-cycle field at $\sim 200 \mathrm{MV} / \mathrm{m}$ that produces a field-driven sideways electron deflection as a function of time ${ }^{21}$. This real-space streaking by a single-cycle field isolates the compressed electron pulses in transverse momentum space with sub-femtosecond precision and therefore provides a direct metrology of the effects of the first interaction's singlecycle control in time.

Figure 1d shows the observed streaking pattern on the screen, plotted as a function of the delay $\Delta t$ between the control field and the streaking field. The largest streaking angle is more than $0.4 \mathrm{mrad}$, corresponding to the absorption or emission of more than 900 photons at $6.9 \mu \mathrm{m}$ central wavelength. The highest streaking speed around $\Delta t \approx \pm 11 \mathrm{fs}$ is $\pm 0.1 \mathrm{mrad} / \mathrm{fs}$, enabling attosecond time resolution ${ }^{13,34}$. There are pronounced streaking oscillations of sideways deflection as a function of delay, but unlike in previous cases with multi-cycle fields ${ }^{13}$ we see here a streaking signal that does not repeat itself before or after one optical cycle of delay. In other words, the streaking peak shapes around $\Delta t \approx 0 \mathrm{fs}$ (peak 0 ; dotted rectangle) and around 
$\Delta t \approx \pm 23$ fs (peaks +2 and -2 ) differ substantially in magnitude and shape. These observations suggest the presence of one exceptional peak of electron density with sub-cycle duration within the pattern of compressed electron density in our beam.

The left panel of Fig. 1e shows a magnified view of the large-angle streaking signal around $\Delta t \approx 0 \mathrm{fs}$ (see dotted rectangle in Fig. 1d). The measured streaking intensity shows a broad maximum at the turning point around $\sim 0.42 \mathrm{mrad}$, a hole with two separate borders in time at $\sim 0.35 \mathrm{mrad}$ (dotted line) and a temporally washed-out pattern at lower angles. Results of a numerical simulation (see Methods) are depicted in the right panel of Fig. 1e for an electron pulse duration of $1.0 \mathrm{fs}$ (full-width-at-half-maximum). A more detailed comparison between experiment and simulations is shown in Fig. 1f, where we plot a cut through Fig. 1e at a deflection angle of $0.37 \mathrm{mrad}$. We see a high streaking intensity at $\Delta t \approx-2 \mathrm{fs}$, followed by a minimum at $\Delta t \approx 0 \mathrm{fs}$ and again a maximum at $\Delta t \approx+2 \mathrm{fs}$. This double peak in time with dip in the middle resembles almost the classical time-dependent deflection dynamics that would occur for electron pulses of negligible duration in time ${ }^{21}$. Comparison of the measured data in Fig. $1 \mathrm{f}$ (black dots) to the results of the simulations (blue lines) indicates an electron pulse duration somewhere between $0.5 \mathrm{fs}$ (dashed blue line) and $1.0 \mathrm{fs}$ (solid blue line).

For a more profound characterization and for understanding the role of potential electron density peaks from adjacent compression cycles (see Fig. 2a), we consider the single-cycle nature of the streaking deflection at high angles (see Fig. 1c) and apply a numerical deconvolution and fitting procedure (see Methods). Basically, streaking angles above $0.35 \mathrm{mrad}$ can only originate from an isolated but finite range of time around $t=0$ with few-femtosecond duration (see Fig. 1c). A numerical consideration of the angular divergence of the electron beam, causing angular blurring in Fig. 1e, provides a temporal instrument response function that we use for deconvolution (see Methods). Figure $2 \mathrm{~b}$ shows the resulting time-dependent electron current density as a function of the peak field strength of the central control cycle. A peaking electron density emerges at points in time where the electric field has positive peaks (dotted lines). There, we have a close-to-linear time-dependent acceleration; preceding electrons are decelerated and trailing electrons are accelerated. In contrast, there is no electron pulse compression for optical cycles with negative field strengths. 
Although the secondary positive field crests (peaks A, B and D in Fig. 2b) can also create a bunched electron current, these cycles have lower field strengths and therefore produce distinguishable compression results. At $\sim 25 \mathrm{MV} / \mathrm{m}$, for example, peak $\mathrm{C}$ is well compressed, but all adjacent peaks are still long. A higher compression strength (lower panels) disperses the central peak towards longer duration and instead compresses the adjacent peaks A, B and D. Figure $2 \mathrm{c}$ shows the retrieved electron pulse durations (see Methods) as a function of the compression strength; the dashed lines denote the results of quantum mechanical simulations ${ }^{35}$. We see that each of the optical cycles produces a minimum electron pulse duration at substantially different compression strength. Exceptional conditions are for example achieved at $\sim 25 \mathrm{MV} / \mathrm{m}$ (third panel of Fig. $2 \mathrm{~b}$ ), where the central electron pulse $\mathrm{C}$ is almost isolated in time (assuming a full-width-at half-maximum criterion), or again at $\sim 80 \mathrm{MV} / \mathrm{m}$, where we observe an over-dispersion (depletion) of peaks B-D but emergence of an isolation of peak A (see Fig. S2). In principle, any optical field cycle of the compression waveform that is unique with respect to the other field cycles can selectively optimize the compression of a single peak density of electrons in time, and subsequent single-cycle time-dependent sideways deflection (Fig. 1d) into a high-angle aperture isolates this pulse from the satellites and time-independent background.

Further evidence for the single-cycle control of our electron beam can be obtained from a scan of the carrier-envelope phase $\phi$ CEP of the compression waveform. Figures $2 \mathrm{~d}$-e show the raw high-angle streaking data at $>0.35 \mathrm{mrad}$ (black dots) and the retrieved electron density (blue) as a function of time for $\phi_{\mathrm{CEP}} \approx 0$ (cosine waveform) and $\phi_{\mathrm{CEP}} \approx \pm \pi$ (minus-cosine waveform); a continuous scan of $\phi_{\mathrm{CEP}}$ is reported in the supplementary materials. With the cosine-shaped modulation field, we create an electron pulse structure with one exceptional peak (upper panels in Figs. 2d-e) but the minus-cosine-like modulation field produces two almost equally high peaks (lower panels in Figs. 2d-e) that originate from the two previously negative field cycles at $\pm 11 \mathrm{fs}$ (see Fig. 2a). Figure $2 \mathrm{f}$ depicts the compressed electron pulse durations as a function of the carrier-envelope phase for the three peaks B-D in Fig. 2d. The middle peak (C) has the shortest duration at $\phi_{\mathrm{CEP}}=0$ (cosine field) while peak B becomes shorter and peak D becomes longer when increasing $\phi_{\mathrm{CEP}}$. At $\phi_{\mathrm{CEP}} \approx \pm \pi$ (minus-cosine field), peaks exchange places (see supplementary materials) and there emerges a double-peak structure (see lower panels in Figs. 
2d-e). We conclude that a simple change of the absolute direction of the control field produces electron current densities of substantially different shape in time.

It is also possible to produce a multi-pulse sequence of few-femtosecond electron pulses with longer mid-infrared pulses (see Methods) in which there are no single-cycle effects. At an optical pulse duration of $\sim 800 \mathrm{fs}$ (see Fig. 3a), the field cycles used for modulation and streaking have approximately equal field strength over the entire duration of the incoming electron pulses from the source ( $\sim 500 \mathrm{fs})$. The observed delay-dependent streaking signal (Fig. 3b) therefore consists of tens of coherent oscillations over hundreds of femtoseconds, demonstrating the creation of multiple electron pulses with sub-cycle duration in synchrony to the optical cycles of the streaking field ${ }^{13}$. Carrier-envelope phase effects are indistinguishable from a delay in this experiment. Figure $3 \mathrm{c}$ shows the evaluated electron pulse duration as an average value over all the individual pulses in the sequence. There is an optimum shortness at $\sim 25 \mathrm{MV} / \mathrm{m}$, similar to the best field strength needed for the single-cycle control (compare Fig. 2b). The shortest average pulse duration of $3.1 \pm 0.7 \mathrm{fs}$ (full width at half maximum) is a little longer than the best singlecycle results ( $\leq 1 \mathrm{fs}$; see Figs. 1f and $2 \mathrm{c}$ ), because inelastic energy losses at the modulation membrane or differences of chirp of the involved laser and electron pulses may contribute to a varying duration of the different individual pulses in the burst. All such effects are irrelevant in case of the single-cycle control. Nevertheless, a cycle-locked few-femtosecond electron pulse train in which the individual pulses are separated by tens of femtoseconds instead of few femtoseconds for near-infrared excitation ${ }^{10,11,13,25-27}$ can be useful for waveform electron microscopy ${ }^{13}$, electron acceleration ${ }^{10,11}$ or quantum control of electron wavepackets ${ }^{14,36}$ in cases where isolated attosecond electron pulses are not necessarily required ${ }^{13}$.

In summary, the reported results demonstrate that the temporal shape of freely propagating high-energy electrons can be controlled and shaped by the field cycles of single-cycle light. Attosecond science with electrons in isolated electromagnetic field cycles is therefore advanced from $\mathrm{eV}$-level energies ${ }^{1-9}$ into the sub-relativistic and relativistic regime of the free-space electron beams. The only prerequisite of our approach is support of at least approximately one optical octave of bandwidth by the modulation element. Sub-cycle optical control of the energy, temporal shape and space-time correlations of beam electrons with attosecond precision will for 
example enable the injection of isolated attosecond electron pulses into laser-driven particle accelerators ${ }^{10,11}$, the attosecond-Angstrom imaging of complex material dynamics with electron microscopy or diffraction ${ }^{13}$, the coherent control of quantum systems ${ }^{37}$ and radiation processes ${ }^{38}$ or the ultrafast modulation and tomography of free-electron quantum states ${ }^{14,17,26}$ in energy and time. More generally, unifying the power and brightness of modern free-space electron beams with the ultimate control of time by modern attosecond science may provide a general novel tool for exploring and controlling complex materials at unprecedented levels of flexibility, power, energy and time.

Acknowledgements. This work was supported by the European Research Council (CoG No. 647771) and the Munich-Centre for Advanced Photonics. We thank Bo-Han Chen and Christina Hofer for helpful discussions on mid-infrared optics, Simon Stork and Jerzy Szerypo for membrane coating, Alexander Gliserin for help with measurement automation and Ferenc Krausz for generous supply of laboratory infrastructure. YM acknowledges general support from Peter Hommelhoff.

Author contribution. YM and PB conceived the experiment. YM performed the experiment and analysed the data. PB and YM discussed the results and wrote the manuscript.

Competing interests. The authors declare no competing interests.

Supplementary information. Supplementary Figs. S1-S3.

Data availability. All relevant data and simulation codes are available from the corresponding authors upon reasonable request. 


\section{References}

1. Paulus, G. G., Grasbon, F., Walther, H., Villoresi, P., Nisoli, M., Stagira, S., Priori, E. \& De Silvestri, S. Absolute-phase phenomena in photoionization with few-cycle laser pulses. Nature 414, 182-184 (2001).

2. Kling, M. F., Siedschlag, Ch., Verhoef, A. J., Khan, J. I., Schultze, M., Uphues, Th., Ni, Y., Uiberacker, M., Drescher, M., Krausz, F., Vrakking, M. J. J. Control of Electron Localization in Molecular Dissociation. Science 312, 246-248 (2006).

3. Krüger, M., Schenk, M. \& Hommelhoff, P. Attosecond control of electrons emitted from a nanoscale metal tip. Nature 475, 78-81 (2011).

4. Piglosiewicz, B., Schmidt, S., Park, D. J., Vogelsang, J., Groß, P., Manzoni, C., Farinello, P., Cerullo, G. \& Lienau, C. Carrier-envelope phase effects on the strong-field photoemission of electrons from metallic nanostructures. Nat. Photonics 8, 37-42 (2014).

5. Putnam, W. P., Hobbs, R. G., Keathley, P. D., Berggren, K. K. \& Kärtner, F. X. Opticalfield-controlled photoemission from plasmonic nanoparticles. Nat. Phys. 13, 335-339 (2017).

6. Rybka, T., Ludwig, M., Schmalz, M. F., Knittel, V., Brida, D. \& Leitenstorfer, A. Subcycle optical phase control of nanotunnelling in the single-electron regime. Nat. Photonics 10, 667-670 (2016).

7. Schiffrin, A., Paasch-Colberg, T., Karpowicz, N., Apalkov, V., Gerster, D., Mühlbrandt, S., Korbman, M., Reichert, J., Schultze, M., Holzner, S., Barth, J. V., Kienberger, R., Ernstorfer, R., Yakovlev, V. S., Stockman, M. I. \& Krausz, F. Optical-field-induced current in dielectrics. Nature 493, 70-74 (2013).

8. Higuchi, T., Heide, C., Ullmann, K., Weber, H. B. \& Hommelhoff, P. Light-field-driven currents in graphene. Nature 550, 224-228 (2017).

9. Schlauderer, S., Lange, C., Baierl, S., Ebnet, T., Schmid, C. P., Valovcin, D. C., Zvezdin, A. K., Kimel, A. V., Mikhaylovskiy, R. V. \& Huber, R. Temporal and spectral fingerprints of ultrafast all-coherent spin switching. Nature 569, 383-387 (2019).

10. Schönenberger, N., Mittelbach, A., Yousefi, P., McNeur, J., Niedermayer, U. \& Hommelhoff, P. Generation and Characterization of Attosecond Microbunched Electron Pulse Trains via Dielectric Laser Acceleration. Phys. Rev. Lett. 123, 264803 (2019).

11. Black, D. S., Niedermayer, U., Miao, Y., Zhao, Z., Solgaard, O., Byer, R. L. \& Leedle, K. J. Net Acceleration and Direct Measurement of Attosecond Electron Pulses in a Silicon Dielectric Laser Accelerator. Phys. Rev. Lett. 123, 264802 (2019).

12. Maroju, P. K. et al. Attosecond pulse shaping using a seeded free-electron laser. Nature 578, 386-391 (2020).

13. Morimoto, Y. \& Baum, P. Diffraction and microscopy with attosecond electron pulse trains. Nat. Phys. 14, 252-256 (2018).

14. Feist, A., Echternkamp, K. E., Schauss, J., Yalunin, S. V, Schäfer, S. \& Ropers, C. Quantum coherent optical phase modulation in an ultrafast transmission electron microscope. Nature 521, 200-203 (2015).

15. Zewail, A. H. Four-Dimensional Electron Microscopy. Science 328, 187-193 (2010). 
16. Miller, R. J. D., Ernstorfer, R., Harb, M., Gao, M., Hebeisen, C. T., Jean-Ruel, H., Lu, C., Moriena, G. \& Sciaini, G. 'Making the molecular movie': first frames. Acta Crystallogr. Sect. A Found. Crystallogr. 66, 137-156 (2010).

17. Echternkamp, K. E., Feist, A., Schäfer, S. \& Ropers, C. Ramsey-type phase control of free-electron beams. Nat. Phys. 12, 1000-1004 (2016).

18. Van Oudheusden, T., Pasmans, P. L. E. M., Van Der Geer, S. B., De Loos, M. J., Van Der Wiel, M. J. \& Luiten, O. J. Compression of subrelativistic space-charge-dominated electron bunches for single-shot femtosecond electron diffraction. Phys. Rev. Lett. 105, $1-$ 4 (2010).

19. Gliserin, A., Walbran, M., Krausz, F. \& Baum, P. Sub-phonon-period compression of electron pulses for atomic diffraction. Nat. Commun. 6, 8723 (2015).

20. Maxson, J., Cesar, D., Calmasini, G., Ody, A., Musumeci, P. \& Alesini, D. Direct Measurement of Sub-10 fs Relativistic Electron Beams with Ultralow Emittance. Phys. Rev. Lett. 118, 154802 (2017).

21. Kealhofer, C., Schneider, W., Ehberger, D., Ryabov, A., Krausz, F. \& Baum, P. Alloptical control and metrology of electron pulses. Science 352, 429-433 (2016).

22. Zhang, D., Fallahi, A., Hemmer, M., Wu, X., Fakhari, M., Hua, Y., Cankaya, H., Calendron, A.-L., Zapata, L. E., Matlis, N. H. \& Kärtner, F. X. Segmented terahertz electron accelerator and manipulator (STEAM). Nat. Photonics 12, 336-342 (2018).

23. Zhao, L. et al. Terahertz Streaking of Few-Femtosecond Relativistic Electron Beams. Phys. Rev. X 8, 021061 (2018).

24. Ehberger, D., Mohler, K. J., Vasileiadis, T., Ernstorfer, R., Waldecker, L. \& Baum, P. Terahertz Compression of Electron Pulses at a Planar Mirror Membrane. Phys. Rev. Appl. 11, 024034 (2019).

25. Sears, C. M. S., Colby, E., Ischebeck, R., McGuinness, C., Nelson, J., Noble, R., Siemann, R. H., Spencer, J., Walz, D., Plettner, T. \& Byer, R. L. Production and characterization of attosecond electron bunch trains. Phys. Rev. Spec. Top. - Accel. Beams 11, 061301 (2008).

26. Priebe, K. E., Rathje, C., Yalunin, S. V., Hohage, T., Feist, A., Schäfer, S. \& Ropers, C. Attosecond electron pulse trains and quantum state reconstruction in ultrafast transmission electron microscopy. Nat. Photonics 11, 793-797 (2017).

27. Kozák, M., Schönenberger, N. \& Hommelhoff, P. Ponderomotive Generation and Detection of Attosecond Free-Electron Pulse Trains. Phys. Rev. Lett. 120, 103203 (2018).

28. Schmid, K., Veisz, L., Tavella, F., Benavides, S., Tautz, R., Herrmann, D., Buck, A., Hidding, B., Marcinkevicius, A., Schramm, U., Geissler, M., Meyer-ter-Vehn, J., Habs, D. \& Krausz, F. Few-Cycle Laser-Driven Electron Acceleration. Phys. Rev. Lett. 102, 124801 (2009).

29. Kozák, M., Förster, M., McNeur, J., Schönenberger, N., Leedle, K., Deng, H., Harris, J. S., Byer, R. L. \& Hommelhoff, P. Dielectric laser acceleration of sub-relativistic electrons by few-cycle laser pulses. Nucl. Instruments Methods Phys. Res. Sect. A Accel. Spectrometers, Detect. Assoc. Equip. 865, 84-86 (2017).

30. Guénot, D., Gustas, D., Vernier, A., Beaurepaire, B., Böhle, F., Bocoum, M., Lozano, M., Jullien, A., Lopez-Martens, R., Lifschitz, A. \& Faure, J. Relativistic electron beams driven by kHz single-cycle light pulses. Nat. Photonics 11, 293-296 (2017). 
31. Chen, B.-H., Wittmann, E., Morimoto, Y., Baum, P. \& Riedle, E. Octave-spanning singlecycle middle-infrared generation through optical parametric amplification in LiGaS2. Opt. Express 27, 21306 (2019).

32. Kirchner, F. O., Gliserin, A., Krausz, F. \& Baum, P. Laser streaking of free electrons at $25 \mathrm{keV}$. Nat. Photonics 8, 52-57 (2013).

33. Vanacore, G. M., Madan, I., Berruto, G., Wang, K., Pomarico, E., Lamb, R. J., McGrouther, D., Kaminer, I., Barwick, B., García de Abajo, F. J. \& Carbone, F. Attosecond coherent control of free-electron wave functions using semi-infinite light fields. Nat. Commun. 9, 2694 (2018).

34. Morimoto, Y. \& Baum, P. Attosecond control of electron beams at dielectric and absorbing membranes. Phys. Rev. A 97, 033815 (2018).

35. Baum, P. Quantum dynamics of attosecond electron pulse compression. J. Appl. Phys. 122, 223105 (2017).

36. Pan, Y., Zhang, B. \& Gover, A. Anomalous Photon-Induced Near-Field Electron Microscopy. Phys. Rev. Lett. 122, 183204 (2019).

37. Gover, A. \& Yariv, A. Free-Electron-Bound-Electron Resonant Interaction. Phys. Rev. Lett. 124, 064801 (2020).

38. Pan, Y. \& Gover, A. Spontaneous and stimulated emissions of a preformed quantum freeelectron wave function. Phys. Rev. A 99, 052107 (2019). 



Figure 1. Single-cycle control of sub-relativistic beam electrons. a. Concept and experiment. A 70-keV electron beams is temporally modulated and analysed by a single optical cycle of a mid-infrared field (red). WP, waveplate; P, polarizer; AP, aperture. b. Spectrum of the mid-infrared pulses. c. Electric field waveform of the mid-infrared pulses. d. Streaking signal of the temporally modulated electron pulses as a function of the modulationstreaking delay $\Delta t$. e. Streaking signal of the central feature (left panel) in comparison to a simulation (right panel) for an electron pulse duration of 1.0 fs (full width at half maximum). f. Slice of the streaking pattern at an angle of $0.37 \mathrm{mrad}$. The observations (black circles) range between a simulation with an electron pule duration of $1.0 \mathrm{fs}$ (blue solid curve) and $0.5 \mathrm{fs}$ (blue dotted curve). 

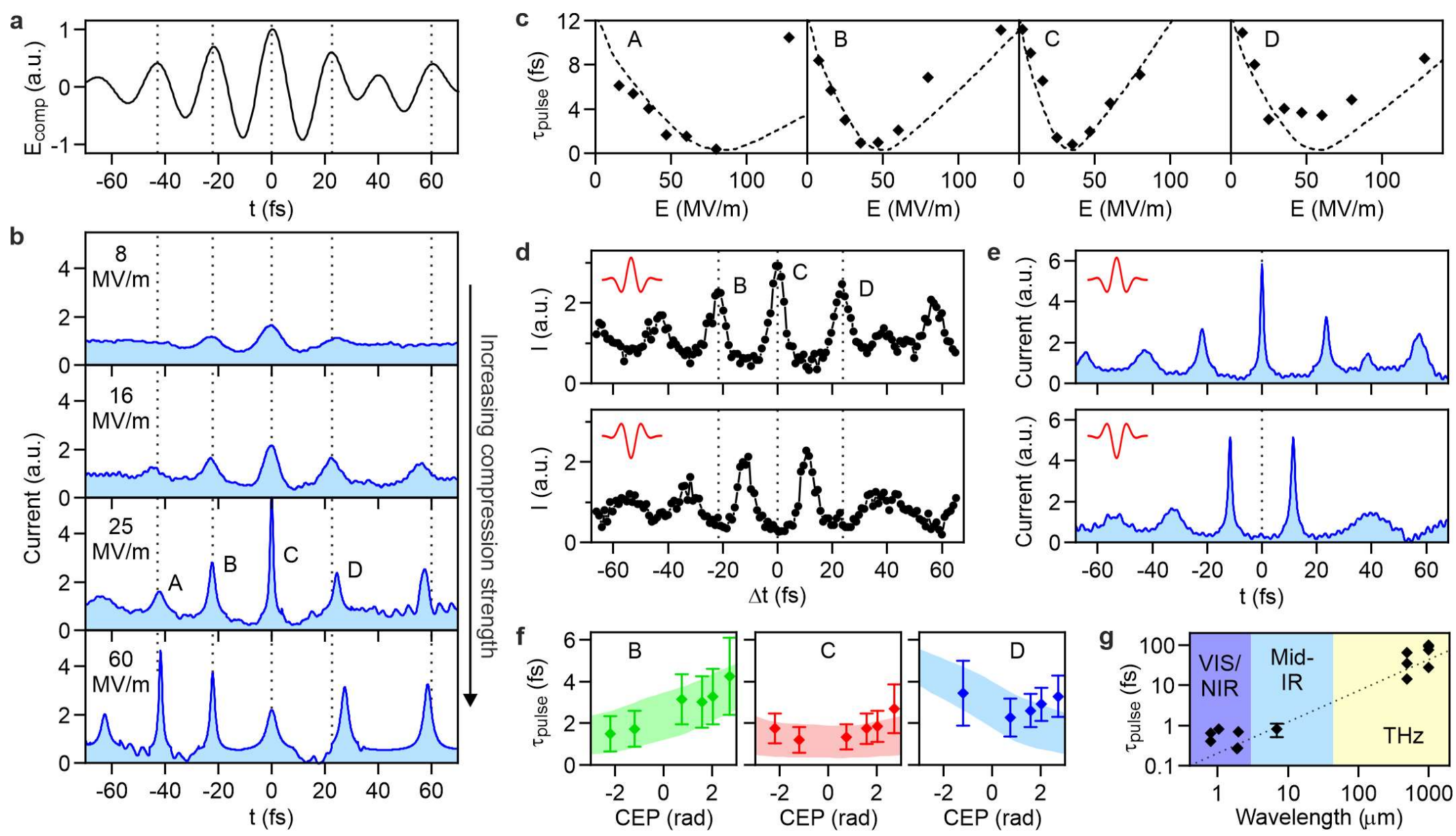

Figure 2. Electron pulse formation and carrier-envelope phase effects. a. Waveform of the modulation field. $\mathbf{b}$. Electron current density in dependence of an increasing modulation strength. c. Electron pulse durations (black squares) for the four peaks A-D (see b) as a function of the field strength of the central cycle. The shortest full-width-at-half-maximum durations are $0.6 \pm 0.6 \mathrm{fs}(\mathrm{A}), 0.9 \pm 0.8 \mathrm{fs}(\mathrm{B}), 0.8 \pm 0.6 \mathrm{fs}$ (C) and $3.1 \pm 1.8 \mathrm{fs}$ (D). Dashed lines depict the result of quantum mechanical simulations. d-e, Carrier-envelope phase control. d. Time-dependent intensity at highest streaking angle ( $>0.35 \mathrm{mrad}$ ) for a cosine field (upper panel) and for a minus-cosine field (lower panel). e. Retrieved electron current density for the two control field shapes. The appearance of a single peak or double peaks is determined by the carrier-envelope phase. f. Pulse durations (dots) of the peaks B-D as a function of the carrier-envelope phase. The transparent bands show the results of simulations with error margins. g. Survey of the electron pulse durations so far obtained at different control wavelengths. Single-cycle electron control is applicable to any wavelength for which an octave-broad modulation element can be designed (see Methods). 

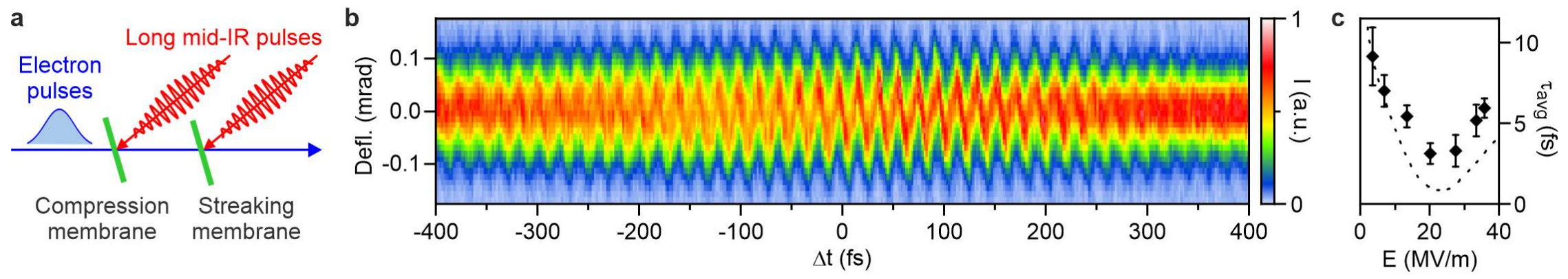

Figure 3. Free-electron control with multi-cycle mid-infrared fields. a. Experimental scheme for multi-cycle mid-infrared control. b. Measured streaking data. The oscillation period and therefore the temporal separation of the electron pulses are $\sim 21 \mathrm{fs}$. c. Train-averaged electron pulse duration (dots) as a function of the compression field strength in comparison to the results of quantum mechanical simulations (dashed line). 